\title{
GEOGRAFÍA Y CARTOGRAFÍA: VÍNCULOS ACTUALES EN APOYO A LA TOMA DE DECISIONES EN EL ORDENAMIENTO TERRITORIAL
}

Gustavo D. Buzai ${ }^{1}$

Resumen: Los vínculos actuales entre Geografía y Cartografía están generados principalmente a través de las tecnologías digitales, de las cuales los Sistemas de Información Geográfica (SIG) ocupan un lugar central al ser considerados el núcleo de la Geoinformática. Los Sistemas de Información Geográfica han producido una innegable revolución tecnológica, pero principalmente han producido una notable revolución intelectual al trasladar conceptos del análisis espacial hacia un contexto de mayor amplitud que el de la Geografía, considerada la ciencia que le dio origen. La revolución tecnológica se encuentra estrechamente ligada con los métodos y técnicas que se han estandarizado para entender los modos de organización del espacio geográfico, en esta línea, los estudios de focalización espacial se vinculan a la cartografía como lenguaje privilegiado que supera ampliamente la condición tradicional del inventario. Por su parte, la revolución intelectual se encuentra relacionada a la forma en que se puede pensar la realidad, es decir, lograr un conocimiento preciso de la base empírica en la cual el ser humano desarrolla sus diversas actividades en el planeta. La vinculación actual entre Geografía y Cartografía supera la manifestación tecnológica para adentrarse en las relaciones teórico-metodológicas que surgen en el marco de la Geografía Aplicable/Aplicada. Sus resultados están claramente dirigidos a la búsqueda de respuestas concretas a problemáticas socioespaciales de diferente naturaleza, pero enmarcadas en un similar contexto: la dimensión espacial. En su interior, las respuestas estarán dirigidas a aspectos que puedan ser comprendidos a través de los conceptos de localización, distribución, asociación, interacción y evolución espacial. Desde esta perspectiva la Geografía es considerada como ciencia de la organización del territorio, la cual enmarcada en acciones voluntarias da lugar al ordenamiento territorial, el cual, realizado por la administración pública incluye dos grandes fases: planificación y gestión. En ambas los SIG y la Cartografía tienen incidencia, aunque desde el ámbito académico su mayor participación estaría dada en la fase de planificación realizando el diagnóstico y generando propuesta en apoyo a la toma de decisiones. La exposición se basará principalmente en la presentación de diferentes procedimientos que ejemplifican lo anteriormente expuesto, como el análisis exploratorio de datos espaciales, modelado cartográfico, evaluación multicriterio y modelos de localización-asignación, entre otros. En síntesis, la relación actual entre Geografía y Cartografía, sintetiza la confluencia entre desarrollos teórico-metodológicos como aportes fundamentales para la resolución de problemáticas socioespaciales concretas en la práctica del ordenamiento territorial.

\footnotetext{
${ }^{1}$ Universidad Nacional de Luján, Programa de Estudios Geográficos. E-mail: buzai@uolsinectis.com.ar; Web: www.gesig-proeg.com.ar
} 


\section{LA BASE EMPÍRICA}

Importantes vínculos actuales entre la Geografía Aplicada y la Cartografía están generados a través de la tecnología de los Sistemas de Información Geográfica (SIG) (Ruiz, 2010), los cuales ocupan un claro lugar central al ser considerados el núcleo de la Geoinformática.

Los Sistemas de Información Geográfica han producido una innegable revolución tecnológica, pero principalmente han producido una notable revolución intelectual. La primera se encuentra estrechamente relacionada con los métodos y técnicas que se han estandarizado para entender los modos de organización del espacio geográfico y actuar en el análisis espacial centrado en la gestión y planificación del territorio, gestión entendida como administración de lo existentes y planificación como tareas prospectivas hacia el logro de un mejor funcionamiento. La segunda se encuentra vinculada a la forma en la que se puede pensar la realidad, es decir, la base empírica en la cual el ser humano ha desarrolla sus diversas actividades en el planeta.

Esta base empírica está constituida por los elementos materiales localizados y se presenta al investigador como un paisaje global formado por tres esferas de diferente potencialidad y ritmos de evolución: una abiótica formada por la geosfera (atmósfera, hidrósfera y litósfera), una biótica formada por los seres vivos que componen la biosfera y una humana compuesta por la sociedad, también denominada esfera de conocimiento o noosfera.

Aunque el hombre también pertenece a la esfera biótica, la separación se realiza principalmente por su componente cultural, porque, mientras la mayoría de los seres vivos tienen como finalidad adaptarse y sobrevivir en el mundo, el hombre como especie es el único que ha tratado de comprenderlo y mediante este intento ha creado un cuerpo de ideas y conceptos que desembocó en la llamada ciencia.

En base a sus características, la ciencia puede definirse como un conocimiento racional, sistemático, que tiende a la exactitud, que es verificable y, que por lo tanto, puede ser falible.

Es racional porque es producto de la razón humana, sale de la mente de los individuos, se rige principalmente por aspectos lógicos e intenta apartarse de las cuestiones emocionales y valorativas en la construcción de conocimiento. Una situación que puede generar tensiones ya que el investigador ha formado un marco epistémico relativo a su espacio-tiempo-sociedad. Es 
sistemático porque no es un conocimiento que se produzca al azar, sino que existen ciertos caminos y reglas para su formulación. Tiende a ser exacto porque cada aproximación a la realidad busca mayor grado de correspondencia entre sus enunciados observacionales y la base empírica del mundo real. Y finalmente puede verificarse, a través de contrastar las hipótesis formuladas, que estas no sean verdaderas. En este sentido recordamos que la verdad, en ciencia, siempre es provisoria.

Cabe mencionar que a diferentes niveles en la profundidad de las investigaciones (exploratoria, descriptiva, clasificatoria o explicativa) los Sistemas de Información Geográfica se han transformado en importantes auxiliares en la generación de hipótesis y sus posibles respuestas, las hipótesis formuladas por oposición o paralelismo pueden resolverse con el Análisis Exploratorio de Datos Espaciales (ESDA), las hipótesis de relación causa-efecto a través del análisis de regresión múltiple ajustada geográficamente (GWR) y las formas recapitulativa o interrogativa son combinaciones de las anteriores. En todas ellas el resultado final se presenta mediante cartografía específica.

\section{Perspectiva sistémica}

La realidad nos brinda estímulos permanentes y muchos de ellos los podemos ir captando con nuestros elementos de medición cada vez más sofisticados. Algunos datos resultan contundentes: cuando se puso en órbita en el año 1967 el satélite artificial para la exploración de los recursos naturales LANDSAT-1 y finalizó su primera órbita completa a la Tierra obtuvo un volumen de información equivalente a la que los geógrafos tenían disponibles hasta el siglo XV y en la segunda órbita elevó el caudal de información llegando a la equivalente en siglo XIX (Stotman, 1999).

Podemos afirmar, sin temor a equivocarnos, que rápidamente esta avalancha de información nos sobrepasó en muchas de nuestras capacidades técnicas, pero no en la del pensamiento.

Tomando una perspectiva de gran amplitud como Macrogeografía, podemos considerar que a través del uso de los satélites también se propició una importante revolución intelectual. Ver la esfera terrestre, como una pequeña bola flotando en el espacio generó un impacto conceptual y ético mayor a lo que originalmente se hubiera esperado (Gould, 1987).

Revista do Departamento de Geografia - USP, Volume Especial Cartogeo (2014), p. 3-22. 
El impacto se refiere a un desplazamiento conceptual de la tradicional postura antropológica etnocéntrica y sociológica de la otredad. Poder ver el planeta Tierra desde el espacio exterior nos hace tomar conciencia del pequeño lugar que ocupamos en el universo y de que todos nos encontramos -como lo decía el astrónomo Carl Sagan (1989)- en el mismo hogar planetario, donde la diferencia entre el yo y el otro, entre nosotros y ellos comienza a perder sentido.

Esta visión planetaria como imagen en movimiento de una esfera azul por los océanos, marrón por los continentes y blanca por el hielo y las nubes, muestra desde un punto de vista empírico la mayor integración del un sistema físico-natural. A esto habría que agregarle la dimensión socio-espacial que también se integra a partir de esa escala global hacia todas las escalas posibles, a través de flujos tangibles e intangibles de infinitas relaciones.

Los estudios correspondientes al cambio climático global, las relaciones económicas internacionales, las relaciones políticas multilaterales y la lucha por el control de los diferentes tipos de flujos (incluyendo los que circulan por el ciberespacio) han tomado un importante protagonismo en el mundo actual. Corresponde a una imagen que nos muestra las más amplias vinculaciones dentro del espacio relacional mundial y de allí hacia diferentes escalas de análisis hasta llegar a los espacios locales y al sitio.

En este punto surge otra tensión en la investigación. Todo lo que estudiamos se encuentra en el espacio geográfico y la investigación debe definirse en algún nivel específico que se encuentra entre los 510 millones de kilómetros cuadrados del planeta y el metro cuadrado que cada uno de nosotros ocupa en cada momento.

Esto nos lleva a pensar en el abordaje de la realidad como totalidad y de allí como sistema. Porque para los que trabajamos en ciencia aplicada, es decir, para los que tenemos intención de utilizar los conocimientos teóricos desarrollados en el ámbito de nuestras ciencias particulares con la finalidad de resolver problemas socio-espaciales, tarde o temprano se nos plantean interrogantes acerca de la manera en la que puede ser captada la realidad.

En esta línea de pensamiento un desarrollo conceptual actual que avanza sobre las características generales presentadas en la Teoría General de los Sistemas propuesta por Von Bertalanffy (1968) se puede encontrar en la Teoría de los Sistemas Complejos basada en la epistemología genética de Jean Piaget (1972) y desarrollada en base a una perspectiva constructivista del trabajo científico interdisciplinario por Rolando García (2006). 
La Teoría de los Sistemas Complejos ha demostrado grandes capacidades para el estudio de la realidad en general. Particularmente he podido comprobar su capacidad, primero, para el estudio de la realidad socio-espacial empírica de la Geografía, y segundo, para conceptuar aspectos específicos correspondientes a la construcción de conocimientos en cuanto a su capacidad epistemológica.

Este mundo global es nuestra realidad empírica de mayor extensión y provee gran cantidad de elementos para su análisis, inclusive en el dominio material de la Geografía. También se considera que la ciencia es empírica porque debe estudiar la materialidad concreta del mundo real y actuar sobre ella.

Considerar la actividad científica -y junto con ella la Geografía- como ciencia empírica no significa avalar el empirismo como perspectiva que considera la objetividad al momento de captar puramente los hechos a través de la experiencia. A través de una postura constructivista queda claro que los sistemas no están definidos pero son definibles, y la construcción sistémica en cualquier escala y extensión se realiza a través de los datos como estímulos generados por la realidad, los observables como datos interpretados por el observador, y los hechos formados por las relaciones entre observables.

Pero lo que deseo resaltar, principalmente de esta perspectiva, corresponde al entendimiento de la realidad como una estructura perteneciente a un universo estratificado. Es decir, la composición de la realidad por niveles de organización semi-autónomos y en los que rigen dinámicas específicas para cada uno de ellos, aunque interactúan entre si.

Esta consideración me ha posibilitado contar con un marco general que permite sustentar la estabilidad de determinadas teorías en niveles específicos y la no-invalidación de ellas a través de consideraciones surgidas de otros niveles de análisis. Pienso que esta consideración sería un complemento de tercera dimensión de la metodología de los Programas de Investigación propuesta por el epistemólogo Imre Lakatos (1983) en el marco del falsacionismo sofisticado, superador del racionalismo crítico del Círculo de Viena. 


\section{Perspectiva geográfica}

Al analizar el pensamiento geográfico, generalmente rescato el concepto de paradigma propuesto por el historiador de la ciencia Thomas Kuhn. Este surge de un modelo que tiene alta correspondencia con el desarrollo de las ciencias duras y permite verificar, aunque con algunos desfasajes, las características que ha tenido la Geografía como ciencia particular.

La definición más usual de paradigma establece que son “...realizaciones científicas universalmente reconocidas que, durante cierto tiempo, proporcionan modelos de problemas y soluciones a una comunidad científica." (Kuhn, 1970), esta consideración, sin dudas nos muestra que la objetividad científica prácticamente no es posible ya que un paradigma no solamente es una estructura que nos permite mirar el mundo de una determinada forma sino que principalmente se encontrará inserta en la legitimidad que puede darle su institucionalización.

La Geografía ha desarrollado a lo largo de su historia diferentes maneras de ver la realidad, las cuales han sido consideradas principalmente como diferentes paradigmas de la Geografía surgidos de cambios revolucionarios. A continuación prestaré principalmente atención a las definiciones producidas a partir de las perspectivas ecológica, corológica y sistémica las cuales interactúan muy estrechamente con el nivel focal de los Sistemas de Información Geográfica y proporcionar las bases de su relación con la Geografía Aplicada.

Podemos mencionar la existencia de un largo período pre-paradigmático en el cual no solamente se llevaron a cabo programas de investigaciones individuales y separados, sino que constituyeron el prolegómeno de una gran crisis científica experimentada por la Geografía a finales del siglo XIX, generada por una gran especialización del conocimiento. La acumulación progresiva de conocimientos brindó objetos de estudios específicos para la aparición de una gran cantidad de nuevas disciplinas denominadas Ciencias de la Tierra que adquirían individualidad separándose de la Geografía como ciencia madre.

Si la Geodesia se ocupaba de las dimensiones terrestres, la Geofísica del campo magnético, la Climatología y la Meteorología se convierten en Ciencias de la Atmósfera, la Oceanografía e Hidrología estudian los cuerpos de agua, la Geología se ocupa del subsuelo a través de la Mineralogía, Estratigrafía, Tectónica, Sismología y Geomorfología, y la Biogeografía se desarrolla como rama de la Biología y aborda el estudio de la evolución de la distribución espacial de 
animales y plantas sobre el planeta; al quedar en evidencia que una única ciencia no podía abarcar todo ese conocimiento, la pregunta es cual debería ser el objeto de estudio de la Geografía para ser definida como ciencia específica y no desaparecer fragmentándose entre diversas disciplinas que abarcaban cada uno de sus contenidos temáticos iniciales.

A finales del siglo XIX el libro Anthropogeografía de Friedrich Ratzel (1882-1891) brindaría una respuesta al incluir al hombre y sus actividades. Por lo tanto, la Geografía, sin dejar de lado su condición naturalista, incluyó definitivamente al hombre y se convertiría en la única disciplina que estudiaría una relación.

Surge una primera definición: La Geografía queda definida desde un punto de vista ecológico como la ciencia que estudia la relación entre el hombre y su entorno, entre la sociedad y el medio. La Geografía encontraba, de esa manera, un lugar en el contexto de las ciencias, pero lo haría como Ciencia Humana.

A pesar de que esta definición puede fecharse a finales del siglo XIX, actualmente desde los Sistemas de Información Geográfica podemos decir que los estudios realizados consideran principalmente las manifestaciones espaciales surgidas de la relación entre la sociedad y su medio.

La Geografía como ciencia humana es la que ha posibilitado mantener una cierta unidad en los estudios geográficos por poco más de cien años. Los geógrafos actuales estamos de acuerdo en que nuestros programas de investigación se apoyarán en el estudio de las relaciones entre la sociedad y el medio, es decir que, si no hay una componente humana no puede haber investigación en Geografía.

Apoyándose en la obra de Bernard Varenius realizada en el siglo XVII (Varenius, 1974) y desarrollando su estudio especial que había quedado inconcluso, se puede considerar que los estudios de Paul Vidal de la Blache (1913), marcan el inicio de la llamada Geografía Regional, perspectiva de análisis que se convertiría en central durante las primera décadas del siglo.

El objeto de estudio de la Geografía Regional es la región. El método de análisis se basa en la descripción porque considera a la región como una realidad objetiva, única e irrepetible. La región es previa al investigador, este debía poder reconocerla y una vez definida se convierte en marco espacial en el cual se deben estudiar las manifestaciones paisajísticas de las 
combinaciones físicas y humanas que de producían en su interior de manera particular y específica.

Mientras las perspectivas geográficas de Friedrich Ratzel y de Paul Vidal de la Blache se basaron filosóficamente en el postivismo de Auguste Comte, aparecería en la misma línea una propuesta de impacto racionalista con el trabajo de Richard Hartsohrne $(1939,1959)$ (postura neokantiana tomada de los trabajos previos de Alfred Hettner) y justificada en la clasificación de las ciencias realizada por Wilhelm Windelband (1970).

Tanto la postura regional como la actualización racionalista consideraban que la Geografía estudia -al igual que la Historia- aspectos únicos e irrepetibles, por lo cual es una ciencia de carácter idiográfico. La diferencia fundamental estaría dada por la característica constructivista que se separa del empirismo. Entonces, para esta nueva postura, la región ya no sería considerada una realidad objetiva, sino que pasaba a ser una construcción racional realizada por el investigador.

El método de superposición de mapas que lleva a la realización de una clasificación espacial desde arriba (de lo general a lo particular) se transforma en un procedimiento central que permite poner límites en el espacio geográfico y definir áreas homogéneas. Aunque estas áreas también eran consideradas únicas e irrepetibles, la posibilidad de construcción fue la base para los siguientes desarrollos.

Surge una segunda definición: La Geografía queda definida desde un punto de vista corológico como la ciencia que estudia la diferenciación de espacios sobre la superficie terrestre.

A pesar de que la definición tuvo aparición a finales de la década de 1930, actualmente desde los Sistemas de Información Geográfica podemos decir que la totalidad de estudios realizados consideran, sin dudas, el estudio de la diferenciación espacial.

El contexto histórico de mediados de siglo, en el cual la segunda guerra mundial ha cumplido un papel preponderante para el desarrollo científico-tecnológico ligado a las actividades militares, luego la necesidad de reconstrucción y posteriormente la carrera espacial, favoreció un gran impulso a las ciencias físico-matemáticas y con ello un importante impacto cuantitativo a las ciencias sociales en general y a la Geografía en particular.

El cambio de visión estaría volcado hacia la búsqueda de un análisis geográfico científico, un nuevo paradigma que desestima la visión idiográfica y se vuelca hacia la generalización como 
método de análisis de una ciencia nomotética (Schaefer, 1953). En el período de ciencia normal dominado por lo que sería considerado un paradigma cuantitativo predominan los estudios en los que se modela la realidad en la búsqueda de leyes científicas.

El abordaje geográfico es principalmente espacial y la región se construye, pero el cambio metodológico establece que esta construcción se produce a partir de la utilización de métodos cuantitativos.

Surge una tercera definición: La Geografía queda definida desde un punto de vista sistémico como la ciencia que estudia las leyes que rigen las pautas de distribución espacial.

Desde un punto de vista sistémico, gran cantidad de estudios realizados con Sistemas de Información Geográfica pueden ser utilizados para corroborar modelos espaciales y tomar referencia de diferentes leyes del comportamiento espacial.

Las posturas enumeradas se han desarrollado durante las primeras siete décadas del siglo veinte. Posteriormente transcurrieron un gran número de sucesos históricos que llevaron a un contexto propicio para la radicalización de las ciencias durante finales de la década de 1960 y principios de la década de 1970 (Peet, 1977), los cuales generaron las perspectivas radicales (radicalmente opuestas al cuantitativismo) en dos vertientes: La Geografía Crítica y la Geografía Humanista.

El paradigma crítico se presenta en favor de una Geografía comprometida para la transformación de la realidad social y, a través de poner en evidencia la crisis de la continua diferenciación producida por el sistema capitalista, intenta ayudar a cambiar el orden establecido. En este sentido se critica la cuantificación como una línea de abordaje que se orienta a apoyar el status quo.

Los estudios basados en el paradigma crítico apuntan al estudio de la relación que existe entre la Geografía y la dominación de clases sociales a partir de las pautas superestructurales en la sociedad capitalista, por este motivo, las formas particulares de esta relación comienzan a ser estudiadas basándose en otras disciplinas, principalmente la economía, sociología y ciencias políticas.

Lo que surge como espacial en este enfoque es la consideración de que el espacio geográfico es un reflejo de las características económicas y políticas más amplias, y que si se quiere modificar la injusticia socio-espacial, habría que actuar en estos niveles porque el espacio geográfico 
quedaría como una dimensión contextual. Una importante cantidad de autores consideran esta situación y muchos, desde las posturas críticas, intentan recobrar la dimensión espacial que quedó disminuida (Baxendale, 2001).

Por otra parte, la postura humanista también se encuentra radicalmente opuesta a la Geografía Cuantitativa, sin embargo, a diferencia del paradigma crítico, esta valoriza aspectos del mundo exterior e interior del ser humano, tales como la percepción, valores y aptitudes hacia el ambiente. La Geografía intentaría comprender estos mundos individuales con categorías idiográficas que no se prestan al análisis científico (RELPH, 1976).

Considerando la Teoría de los Sistemas Complejos, la totalidad de estas líneas de abordaje propias de diferentes paradigmas, que han evolucionado durante poco más de un siglo, pueden ser comprendidas en el marco de un universo estratificado, en el cual cada una de ellas se refiere a una escala de análisis diferente, pero con vínculos, a veces contradictorios y en determinadas ocasiones complementarios.

\section{Geografía Aplicada}

Resulta entonces que considerar a la Geografía como ciencia aplicada implica tener en cuenta que incluye todas las características enunciadas con anterioridad como específicas del conocimiento científico en general, ante la necesidad de aplicar el método científico en la búsqueda de generalidades y regularidades que le permitan explicar y predecir patrones espaciales. En este marco se constituye la Geografía Aplicada, entendida desde un punto de vista amplio como la aplicación de conocimientos y habilidades geográficas para la resolución de problemas sociales, económicos y ambientales (Pacione, 1999).

La Geografía como ciencia aplicada utiliza (o puede utilizar) conocimientos obtenidos a través de las investigaciones básicas no con el objetivo de generar nuevos conocimientos, sino principalmente para aplicar conocimientos que sean útiles a la sociedad.

En síntesis, la investigación básica tiene por finalidad acrecentar los conocimientos teóricos para el progreso de la ciencia y no se preocupa por sus posibles aplicaciones. La investigación aplicada busca conocer para hacer, para actuar, para construir y para modificar: avanza en cuestiones prácticas de aplicación y no para el desarrollo de teorías de valor universal. 
Para lograr sus objetivos de aplicación puede verse a la ciencia como proceso de construcción de conocimientos. Corresponde a una trasformación secuencial entre dos estados centrales: el surgimiento de un problema y las acciones realizadas para encontrarle solución.

Entre ambos estados la ciencia aplicada presenta una serie de fases secuenciales con diferentes componentes integrantes (Buzai, Baxendale y Cruz, 2009).

Como ciencia aplicada de carácter empírico el proceso de investigación lleva a salir desde la realidad a partir del problema para volver a la realidad con propuestas para su solución atravesando fases de tipo conceptual, conceptual-metodológica, metodológica-técnica, de validación y transferencia.

Particularmente en el caso de la transferencia, mediante la realización de diferentes productos, se llega a dos destinos principales: una transferencia hacia la investigación y docencia, y una transferencia hacia la gestión y planificación.

La primera se realiza hacia la zona teórica, prácticas geográficas y los antecedentes (estado de la cuestión) de la investigación. La segunda se dirige hacia la base empírica en estrategias de competencia espacial como la eficiencia buscada principalmente por la actividad privada y la búsqueda de justicia espacial como la equidad necesaria en los servicios públicos.

\section{Ordenamiento territorial}

Existe una cuarta forma de definir a la Geografía, la cual posibilita vincular las tres definiciones operativas analizadas anteriormente. Corresponde a considerarla como ciencia de la organización del territorio.

Esta perspectiva proporciona elementos de gran importancia al momento de actuar concretamente en la resolución de problemáticas socio-espaciales que se presentan en la realidad. Toma una posición que le permite, mediante el trabajo empírico, combinar y sintetizar diferentes perspectivas teóricas de la Geografía en la construcción de conocimientos amplios que posibiliten diagnosticar y realizar propuestas de solución.

Ambas acciones se presentan como componentes de base tecnológica-científica en el interior de la planificación territorial y para realizarlas se han utilizado tradicionalmente herramientas metodológicas de probada aptitud, como el uso de cartografía, sistematización matricial, 
aplicación estadística, entre otras. Los Sistemas de Información Geográfica (SIG), desarrollados en el ámbito de la Geografía, ocupan actualmente un lugar preponderante al ingresar en el ámbito de la planificación vinculando tecnologías existentes y ampliando sus posibilidades a través de la incorporación de conceptos y métodos geográficos aplicados en el análisis espacial de contexto digital.

Como actividad de carácter aplicado, el Ordenamiento Territorial presenta un componente científico asociado al uso de conocimientos, metodologías y herramientas para el análisis territorial y un componente profesional en el que se plasman legalmente una serie de normativas y prácticas orientadas a actuar sobre las estructuras territoriales siguiendo una directriz política. La Geografía como ciencia provee un importante contenido al primer componente y puede apoyar conceptualmente al segundo.

En cuanto a su operatividad concreta, asociada con esta clasificación, es posible diferenciar en el interior del Ordenamiento Territorial una serie de componentes vinculados con actividades de carácter secuencial: la planificación y la gestión, cada uno con sus propios componentes.

$Y$ desde una perspectiva geográfica, se recorre un camino desde el diagnóstico hacia las propuestas donde los Sistemas de Información Geográfica pueden aportar en varias líneas de trabajo, en aplicaciones raster desde la realización de procedimientos de reclasificación hasta la modelización mediante autómatas celulares, y en vectorial desde la realización de cartografía temática hasta el cálculo de regresión múltiple ajustada geográficamente.

Estos procedimientos se presentan como base para el logro de la visión de síntesis hacia el logro de diagnósticos integrados en la vinculación del espacio absoluto y relativo, y en el logro de mapas de limitantes y potencialidades.

\section{Núcleo geográfico y campos emergentes}

Actualmente es posible abordar una realidad socio-espacial lo más completamente posible a partir de considerar cuatro posturas que se desarrollan y corren de forma paralela en el desarrollo conceptual de la Geografía actual: la Geografía de la Percepción (vinculada al paradigma humanista), la Ecología del Paisaje (vinculada a los paradigmas regional y racionalista) (Naveh y Lieberman, 1984), la Geografía Automatizada (vinculada al paradigma 
racionalista y cuantitativo) (Dobson, 1983) y la Geografía Postmoderna (vinculada al paradigma crítico) (Soja, 1989).

Son campos definidos durante la década de 1980 y actualmente presentan una situación verdaderamente sorprendente, por la vitalidad de cada línea y por las variadas y múltiples relaciones surgidas entre ellas (Haggett, 2008).

Analizando el lugar que cada una ocupa, podemos decir que la Geografía Automatizada, como sistematización digital de la postura racionalista y cuantitativa, es aquella que tiene como nivel de focalización el estudio del espacio geográfico contemplando el abordaje simultáneo de la relación entre la sociedad y el ambiente, de la diferenciación espacial y de las leyes del funcionamiento espacial. Los Sistemas de Información Geográfica constituyen una herramienta teórico-metodológica fundamental del análisis del mundo real en este nivel.

Permiten principalmente una focalización estrictamente espacial, y de la misma forma que la matriz de datos geográfica propuesta por Brian J.L. Berry (1964) presenta una síntesis de las perspectivas regional, general e histórica (según la forma en que sea abordada la matriz), los Sistemas de Información Geográfica han hecho operativo y sintetizaron, teórica y metodológicamente, los cinco conceptos fundamentales de naturaleza espacial en que basan las aplicaciones realizadas.

Estos conceptos son los de Localización como ubicación espacial de las entidades geográfica en el espacio absoluto, Distribución como la forma en que se reparten las entidades geográficas, Asociación como la forma en que diferentes entidades localizadas y distribuidas se vinculan con relaciones de tipo vertical u horizontal, Interacción como la forma en que se producen vínculos horizontales que privilegian los movimientos sobre el espacio geográfico en un espacio relativo, y Evolución en la incorporación de la dinámica temporal que permite ver como las entidades geográfica cambian sus pautas espaciales a través del tiempo.

Esto significa que cuando se utiliza un Sistema de Información Geográfica, las aplicaciones estarán apoyadas siempre en diferentes combinaciones de estos conceptos, dependiendo del tipo de análisis que se esté realizando.

Presentamos un ejemplo de Geografía Aplicada y Cartografía con Sistemas de Información Geográfica en el cálculo de la pérdida de suelos productivos por el crecimiento de la Aglomeración Gran Buenos Aires (AGBA). La Figura 1 presenta tres capas temáticas de 
diferentes años, de izquierda a derecha, 1914, 1960 y una proyección de la mancha urbana hacia el 2011. La base de datos contiene capas temáticas originales correspondientes a la configuración espacial de la aglomeración en los años censales argentinos (período 1869-2001). Apelando al concepto de evolución espacial y mediante la modelización por autómatas celulares se obtuvo el resultado a partir de las configuraciones de los años 1991 y 2001 . La Figura 2 presenta tres capas temáticas de diferentes variables, de izquierda a derecha, la primera corresponde al mapa de suelos del área de estudio, la segunda a la cobertura proyectada obtenida a partir del procedimiento anterior y la tercera, apelando al concepto de asociación espacial, la superposición de ambas. El resultado permite calcular cuanta superficie de los diferentes suelos se encuentra inutilizada al haber sido cubierta por la mancha urbana. Los resultados forman parte de un reciente proyecto de análisis de cambios en los usos del suelo (Baxendale y Buzai, 2011).

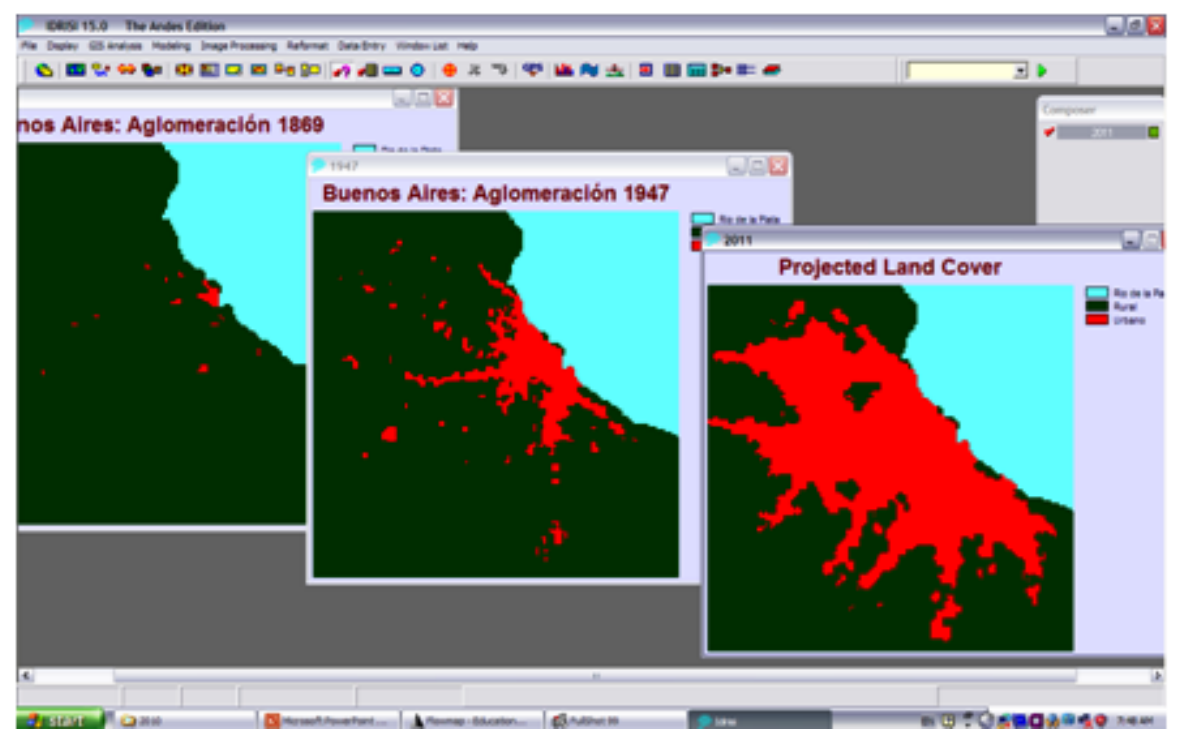

Figura 1: Aglomeración de Buenos Aires. Evolución espacial y proyección al 2011. Fuente: el autor (software IDRISI) 


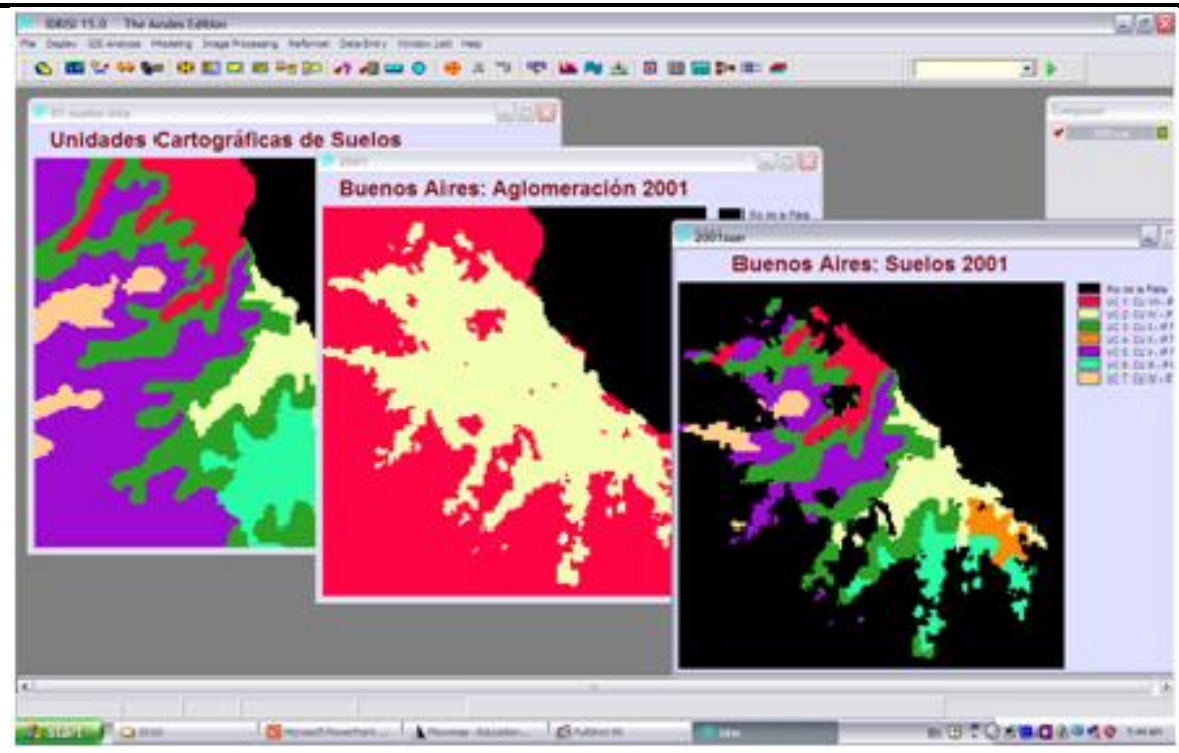

Figura 2: Aglomeración de Buenos Aires. Asociación espacial en el cálculo de la pérdida de suelos productivos 2011. Fuente: el autor (software IDRISI).

Aunque es fundamental destacar, que la importancia de los Sistemas de Información Geográfica no queda únicamente circunscrita al nivel teórico-metodológico disciplinario, sino que avanza hacia otros niveles a causa de su propia naturaleza y del interés demostrado por diversas disciplinas que intentan incorporar la dimensión espacial. Esto último no impacta directamente desde la Geografía como ciencia, sino a partir de la Geografía incorporada en los Sistemas de Información Geográfica que actúa como una notable herramienta generalizada de difusión.

Como hace un siglo la Geografía brindaba objetos de estudio para la aparición de nuevas ciencias, actualmente brinda conceptos y métodos, para que diferentes disciplinas cuenten con una visión más completa de la realidad, una visión que incorpore el espacio geográfico y sus propias estructuras de funcionamiento. Aparece lo que he llamado una Geografía Global (Buzai, 1999) como un paradigma basado en las tecnologías geodigitales, que no representan un paradigma de la Geografía, sino un paradigma geográfico, una visión que nuestra ciencia brinda, a través de los Sistemas de Información Geográfica, a múltiples ciencias y posibilita gran variedad de aplicaciones.

A partir de allí han hecho aparición ciertos campos temáticos de nivel disciplinario (Geografía Automatizada (Dobson, 1983) / Cibergeografía (Dodge y Kitchin, 2001)), interdisciplinario (Geoinformática (ITC, 1991) / Geografía Global (Buzai, 1999)) y transdisciplinario 
(Geocomputación (Longley, Goodchild, Maguire y Rhind, 2001) / Ciencias de la Información Geográfica (Goodchild, 1992) / Ciencias Sociales Integradas Espacialmente (Goodchild y Janelle, 2004) / Neogeografía (Turner, 2006) / Ciencias Geoespaciales (Berry, Griffith y Tiefelsdorf, 2008)). En las perspectivas intedisciplinarias la Geografía se presenta como ciencia de posición central pero nuevamente, luego de un siglo, corre ciertos riesgos. Esta situación brinda una línea de reflexión que lleva a analizar el futuro de la Geografía como ciencia (Buzai, 2007), pero no deja dudas de la importancia que la dimensión espacial ha adquirido en la ciencia actual, y junto a esto, de forma operativa teórico-metodológica que proporcionan los Sistemas de Información Geográfica.

\section{Sistemas de Información Geográfica en la Geografía Aplicada. Evaluación sintética}

De esta manera, una síntesis del rol de los Sistemas de Información Geográfica en la investigación de la Geografía Aplicada actual podría:

$\checkmark$ Considerar la amplitud de estudios posibles en cuanto a la gran variedad de temáticas y procesos en conexiones multiescalares desde el mundo (espacio global) hasta el sitio (espacio local), realizando estudios empíricos constructivistas en donde se enfoque la materialidad espacial concreta de las entidades localizadas sobre el territorio.

$\checkmark$ Considerar la perspectiva sistémica como marco general de análisis, teniendo en cuenta los aportes de la Teoría General de los Sistemas y la Teoría de los Sistemas Complejos en sus potencialidades para el análisis espacial actual. Esto permitiría no solamente verificar aquellas características que se manifiestan de forma conjunta en diferentes escalas, sino principalmente las características propias de cada escala en la consideración de una realidad estratificada de niveles semi-autónomos.

$\checkmark$ Considerar la riqueza conceptual de la Geografía como ciencia madre de los Sistemas de Información Geográfica, tanto en el nivel de focalización espacial apoyado por las perspectivas racionalista y cuantitativa, sino también por la combinación pluriparadigmática hacia niveles de análisis infra-y supra-focal, todo ello en correspondencia con la consideración de la realidad como sistema complejo. 
$\checkmark$ Considerar que esta riqueza conceptual (sumada a la de otros campos disciplinarios) es la que brindará la mejor posibilidad de uso de los Sistemas de Información Geográfica, de los Sistemas de Ayuda a la Decisión Espacial y del proceso de verticalización que los Sistemas de Información Geográfica experimentan actualmente en su desarrollo (Eastman, 2007), comprendiendo que el mejor uso de esta tecnología no se hará a partir de estudiar el manual del usuario, sino que se les podrá sacar verdadero provecho a partir de estudiar Geografía.

$\checkmark$ Considerar que estas temáticas específicas tienen una vocación decididamente empírica, porque las aplicaciones siempre surgen del análisis de la realidad y deben volver a ella para actuar en la búsqueda de soluciones concretas a problemáticas socioespaciales. Los resultados obtenidos siempre pertenecen, académicamente hablando, a una Geografía Aplicable con muy buenas posibilidades de llegar a una Geografía Aplicada (Phlipponneau, 2001) a partir de la fase de transferencia.

$\checkmark$ Considerar que cuando la ciencia se define en cuanto a un propósito los Sistemas de Información Geográfica realizan ciencia aplicada porque corresponde a la aplicación práctica para la resolución de problemas específicos, cuando se define por una estrategia de investigación apoyan una investigación teórica porque se contrasta los resultaos obtenidos con modelos y leyes científicas existentes para comprobarlas o proponer otras, y finalmente, si se define en base al conocimiento a obtener, apoyan una investigación exploratoria en la búsqueda de conocimientos generales de funcionamiento socio-espacial, descriptiva cuando se determina como se producen las diferentes características socio-espaciales y explicativa cuando se llega al porqué o causas de la problemática en estudio (Buzai y Baxendale, 2006).

\section{CONSIDERACIONES FINALES}

Para finalizar debemos mencionar que la sigla SIG ha tenido énfasis en la S (Sig) durante las décadas de 1960 y 1970 porque los iniciales problemas a resolver por aquellos años fueron los de Software, durante las décadas de 1980 y 1990 han tenido énfasis en la I (slg) porque los principales problemas estuvieron centrados a la gestión de la información, mientras que en la 
década del 2000 el énfasis se puso en la G (siG) porque la clave actual está en la Geografía, es decir, en las teorías espaciales que permitan guiar las investigaciones y comprender mejor los resultados obtenidos (Buzai, 2005).

Por lo tanto, no cabe dudas de que los Sistemas de Información Geográfica no solo son una herramienta metodológica, sino principalmente actúan como una herramienta de recorrido conceptual en una gran cantidad de niveles de análisis. A partir de su estrecha relación con la cuantificación, el racionalismo y una perspectiva sistémica generan una base sólida que apoya a las decisiones socio-espaciales con fundamentos científicos de una Geografía Aplicada.

La utilización de un Sistema de Información Geográfica con sus grandes potencialidades (internas $\mathrm{y}$ externas) nos permiten comprender $\mathrm{y}$ actuar sobre la realidad, poniendo a disposición el conocimiento geográfico en el marco de una ciencia aplicada basada en el uso de los Sistemas de Información Geográfica, es un reto para los geógrafos del siglo XXI, que todo este conocimiento se oriente claramente hacia la construcción de un mundo que consiga mayor equilibrio, solidaridad y justicia.

\section{BIBLIOGRAFÍA}

Baxendale, C.A. 2000. Geografía y Planificación Urbana y Regional: una reflexión sobre sus enfoques e interrelaciones en las últimas décadas del siglo XX. Reflexiones Geográficas. 9:5870.

Baxendale, C.A.; Buzai, G.D. 2011. Crecimiento urbano y pérdida de suelos productivos en la aglomeración Gran Buenos Aires (1869-2011), Argentina. En: Buzai, G.D.; Baxendale, C.; Cacace, G.; Dzendoletas, M.A. 2011. Análisis de usos del suelo urbano y regional. Localizaciones óptimas y conflictivas con Sistemas de Información Geográfica. PROEG-11 - UNLU. Luján. pp. 116-132.

Berry, B.J.L. 1964. Approaches to Regional Analysis: A Synthesis. Annals of the Association of American Geographers. 54:2-11.

Berry, B.J.L.; Griffith, D.A.; Tiefelsdorf, M.R. 2008. From Spatial Analysis to Geospatial Science. Geographical Analysis. 40:229-238.

Buzai, G.D. 1999. Geografía Global. Lugar Editorial. Buenos Aires. (2 ${ }^{\text {da }}$. Ed. 2004).

Buzai, G.D. 2005. Geografía Automatizada, Ciencias de la Información Geográfica y Ciencias Sociales Integradas Espacialmente. Avances cuantitativos para los estudios territoriales del siglo XXI. Fronteras. 4(4):31-36. 
Buzai, G.D. 2007. Dilemas de la relación Geografía-SIG entre la disciplina, la interdisciplina y la transdisciplina. Geofocus-Revista Internacional de Ciencia y Tecnología de la Información Geográfica (Editorial). 7:5-7. www.geo-focus.org

Buzai, G.D.; Baxendale, C.A. 2006. Análisis Socioespacial con Sistemas de Información Geográfica. Lugar Editorial. Buenos Aires. (2 ${ }^{\text {da }}$. Ed. Tomo 1, 2011).

Buzai, G.D.; Baxendale, C.; Cruz, M.R. 2009. Fases de un proyecto de investigación en estudios de Geografía Aplicada basados en el uso de Sistemas de Información Geográfica. Fronteras. 8:31-40.

Dobson, J.E. 1983. Automated Geography. The Professional Geographer. 35(2):135-143.

Dodge, M.; Kitchin, R. 2001. Mapping Cyberespace. Routledge. London.

Eastman, R. 2007. La verticalización de los SIG. En: Buzai, G.D. (comp.) Memorias de la XI Conferencia Iberoamericana de Sistemas de Información Geográfica. Universidad Nacional de Luján - SIBSIG. Luján. pp. 183-195.

García, R. 2006. Sistemas Complejos. Conceptos, método y fundamentación espistemológica de la investigación interdisciplinaria. Gedisa. Barcelona.

Goodchild, M.F. 1992. Geographical Information Science. International Journal of Geographic Information Systems. 6(1):31-45.

Goodchild, M.F.; Janelle, D.G. (Eds.) 2004. Spatially Integrated Social Sciences. Oxford University Press. Oxford.

Gould, P. 1987. Pensamientos sobre la Geografía. GeoCrítica. 68:1-39.

Haggett, P. 2008. The Spirit of Quantitative Geography. Geographical Analysis. 40:226-228.

Hartshorne, R. 1939. The Nature of Geography: A critical survey of current thought in the light of the past. Annals of the Association of American Geographers. 29:173-658.

Hartshorne, R. 1959. Perspectives on the Nature of Geography. Rand McMillan. Chicago.

ITC. 1991. Geoinformatics. Programme of Courses. International Training Center. International Institute for Aeroespace Survey and Earth Sciences. Enchede.

Kuhn, T.S. 1970. The Structure of Scientific Revolutions. The Chicago University Press. Chicago.

Lakatos, I. 1983. La metodología de los programas de investigación científica. Alianza. Madrid.

Longley, P.; Goodchild, M.; Maguire, D.; Rhind, D. (eds.) 2001. Geocomputation: A Primer. Wiley. New York. 
Naveh, Z.; Lieberman, S. 1984. Landscape Ecology: Theory and Applications. Springer-Verlag. New York.

Pacione, M. 1999. In pursuit of useful knowledge: the principles and practice of Applied Geography. In: Pacione, M. (Ed.) Applied Geography: Principles and Practice. Routledge. London. pp. 3-18.

Peet, R. 1977. The development of radical geography in the United States. Progress in Human Geography. 1(2):240-263.

Piaget, J. 1972. Psicología y Epistemología. Emecé Editores. Buenos Aires.

Phlipponneu, M. 2001. Geografía Aplicada. Ariel. Barcelona.

Ratzel, F. 1882. Anthropogeographie. Vol.1. Grundzüge der Anwendung der Geographie auf die Geschichte. 1891. Vol.2. Die Geographische Verbreitung des Meschen. Engelhorn. Stuttgart.

Relph, E. 1976. Place and Placeness. Pion. London.

Ruiz, E. 2010. El impacto de las tecnologías de la información geográfica en la Cartografía y la Geografía: Reflexiones sobre 20 años de Sistemas de Información Geográfica. GeoSIG. 2(2):200209 [on-line: www.gesig-proeg.com.ar]

Sagan, C. 1980. Cosmos. The Scott Meredith Literary Agency. New York.

Schaefer, F.K. 1953. Excepcionalism in Geography: A methodological examination. Annals of the Association of American Geographers. 43:226-249.

Soja, E. 1989. Postmodern Geographies: the reassertion of space in critical social theory. Verso. London.

Stotman, J. 1999. Conferencia. Congreso Internacional sobre la Enseñanza de la Geografía frente a un mundo en cambio. Universidad Nacional de Cuyo. Mendoza. 19 al 24 de abril.

Turner, A.J. 2006. Introduction to Neogeography. O’Reilly Media Inc.

Varenius, B. 1974. Geografía General en la que se explican las propiedades generales de la Tierra. Ediciones de la Universidad de Barcelona. (Original publicado en Leyden, 1650).

Vidal de la Blache, P. 1913. Des caracteres distinctifs de la Geographie. Annales de Géographie. 22(124):289-299.

Von Bertalanffy, L. 1968. General Systems Theory: Foundations, Development, Applications. George Braziller. New York.

Windelband, W. 1970. Historia General de la Filosofía. El Ateneo. Buenos Aires. 